\title{
MICROHONGOS ANEMOFILOS EN AMBIENTES ABIERTOS DE DOS CIUDADES DEL NOR- DESTE ARGENTINO
}

\section{(Anemophilous microfungi in outdoor environments of two cities in Argentinian northeastern)}

Palabras claves: Microhongos anemófilos, ambientes abiertos, Argentina Key words: Anemophilous microfungi, outdoor environment, Argentina

RESUMEN

Con el objetivo de estudiar los microhongos anemófilosde las ciudades de Resistencia y Corrientes, ubicadas en el nordeste argentino, se muestrearon por la técnica de depositación gravitacional, dos zonas en cada ciudad. El muestreo se realizó en primavera y otoño, en dos horarios diferentes.

Existen diferencias entre los aislamientos de ambas ciudades. En Resistencia se observó un mayor número de taxa (323) que en Corrientes, (248)siendo más notoria esta diferencia en otoño junto a una mayor diversidad de géneros (34/28). Los géneros más frecuentes en ambas ciudades fueron: Cladosporium, Aspergillus, Penicillium, Phoma,Fusarium, Alternaria, Curvularia y Trichoderma.

Un buen número de los hongos aislados son de interés en salud pública por sus capacidades oportunistas en diversos cuadros clínicos, asi como en alergias o micotoxocosis.

\section{INTRODUCCION}

Cuando se alude a la diversidad biológica, viene a la mente sólo la multiplicidad de plantas y de animales, sin considerar a los microorganismos, quienes representan, en buena medida, gran parte de esta diver-sidad. En el ambiente aéreo se encuentran bacterias, hongos, algas y protozoarios, siendo los dos primeros grupos los predominantes. Estos organismos utilizan diversas

\author{
Patricia Esquivel*, Magdalena Mangiaterra*, \\ Gustavo Giusiano*, María A. Sosa*. \\ Departamento de Micología. Instituto de Medicina Regional. \\ Universidad Nacional del Nordeste Av. Las Heras 727. 3500 \\ Resistencia (Chaco) - Argentina \\ E-mail:paraluchi@hotmail.com
}


el sustrato orgánico, la estación del año, la hora del día, el grado de urbanización y de las especies, entre otras variables $(1,2)$.

Los propágulos de dispersión aéreos se encuentran en la atmósfera que envuelve al planeta desde el polo Sur al Norte, en praderas templadas, selvas tropicales o desiertos. Existe una importante variabilidad cualicuantitativa en la micota anemófila de los distintos ambientes, así como en las de un mismo lugar si se las estudia en diferentes épocas del año. En este sentido influyen definitivamente factores tales como la velocidad de los vientos y su dirección, la temperatura, la humedad y tipo de vegetación del área. El conocimiento de la carga fúngica del aire y su variación local, tanto diaria como estacional, es una importante información para la interpretación de numerosos problemas en patología vegetal, humana y animal. También es útil en el ámbito de la industria alimenticia principalmente en las etapas de manufactura y almacenamiento (1).

Poco se sabe de la potencialidad alergénica de las diversas partículas contaminantes del aire en los ambientes externos y de los niveles de exposición a que deben estar sometidos los pacientes para que se les manifieste una respuesta clínica. En relación a los hongos, su rol como productores de alergias no tiene discusión, siendo conocido también, su papel como agentes etioló-gicos de otomicosis, keratomicosis, bronquitis crónicas, enfisema y asma, entre otras patologías. Cerca de 80 especies han sido asociadas con cuadros de asma, rinitis y/o alergia respiratoria. La sensibilización a una sola especie es muy rara, lo habitual es, según resultados de pruebas cutáneas, se deba a varios hongos, debida a la presencia de epítopes comunes en géneros filogené-ticamenta relacionados $(3,4,5,6)$.

La calidad del aire es un factor importante para la salud ambiental y esta calidad está relacionada, en parte, por la carga fúngica presente (1).

El objetivo de este trabajo fue estudiar la micota anemófila de dos ciudades del nordeste argentino.

\section{MATERIALES Y METODOS}

Se estudiaron los hongos anemófilos de dos ciudades del nordeste argentino, Resistencia ubicada a los $58^{\circ} 51^{\prime}$ longitud oeste y $27^{\circ} 27^{\prime}$ latitud sur y Corrientes a los $58^{\circ} 49^{\prime}$ longitud oeste y $27^{\circ} 27^{\prime}$ latitud sur.

\section{Fisiografía de las ciudades Corrientes y Resistencia}

En el norte argentino se han distinguido dos grandes unidades geográficas: el Chaco y la Mesopotamia, separados por la falla por donde surca el Río Paraná que divide el elevado bloque mesopotámico de la cuenca sedimentaria chaqueña, contribuyendo a la creación de ambientes más o menos individualizados.

En las proximidades de la confluencia de los ríos Paraná - Paraguay se localizan Resistencia y Corrientes. Resistencia está emplazada en el codo externo del Paraná, sobre una planicie aluvial, en el interfluvio de los riachos Negro y Arazá, salpicada de lagunas semilunares y sometida a inundaciones periódicas que cubren un amplio valle de pajonales y palmerales, atravesados por finas galerías boscosas $(7,8)$.

Corrientes está situada en el codo interno, sobre la alta barranca mesopotámica. El paisaje se caracteriza por suaves lomadas arenosas y amplios valles que canalizan los excesos de agua hacia el Paraná. La vegetación dominante es la pradera, especialmente sobre las superficies de mediana altura y las isletas arbóreas que salpican las lomadas, mientras que en los terrenos bajos dominan la paja brava, la totora y los juncos $(7,8)$. Resistencia y Corrientes están ubicadas en una zona subtropical templada sin estación seca. La época de mayores lluvias es entre octubre y abril, siendo los meses más lluviosos noviembre y marzo, a su vez, los más secos son julio y agosto. El promedio anual de lluvias es cercano a los $1300 \mathrm{~mm}$. En Resistencia la temperatura media anual es de $21,7^{\circ} \mathrm{C}$, mientras que en Corrientes es de $20^{\circ} \mathrm{C}$. En ambas ciudades se dan variaciones extremas llegando a los $45^{\circ} \mathrm{C}$ en verano y $0^{\circ} \mathrm{C}$ en invierno (8).

\section{Toma de muestra}

El aire de Corrientes y Resistencia fue muestreado durante los meses de primavera del año 2000 y de otoño del año 2001. Al no tener referencias bibliográficas sobre el universo de hongos del aire en estas zonas, se estimó el número de muestras de acuerdo a la población de cada una de las ciudades muestreadas y la prevalencia de hongos anemófilos hallada en otras ciudades donde se hicieron estudios semejantes $(3,4,9,10,11)$.

El método utilizado fue el de depositación gravitacional horizontal sobre placas de Petri con agar papa dextrosa con antibiótico (cloranfenicol 250mg/l). La placas fueron colocadas aproximadamente a 1,50 m del suelo y abiertas durante 10 minutos.

En cada ciudad se seleccionaron dos lugares, uno céntrico muy transitado y con poca vegetación (Zona 1) y otro residencial, con poco movimiento vehicular y abundante vegetación (Zona 2), separados aproximadamente por 4,3 kilómetros.

Se tomaron muestras cada 15 días durante los tres meses de cada estación. Los horarios de muestreos fueron: por la mañana entre las 7 y las 8 hrs (hora 1) y al mediodía entre las 12 y las 13 hrs (hora 2 ). En cada muestreo se expusieron 3 placas en cada horario y en cada Zona de ambas ciudades, lo que completa un total de 24 placas por 
Tabla 1.- Clasificación de Yadav y Madelin

\begin{tabular}{|c|c|c|}
\hline \multicolumn{3}{|c|}{ Clasificación de Yadav y Madelin } \\
\hline & Categoría & $\begin{array}{l}\text { Frecuencia de } \\
\text { aparición }\end{array}$ \\
\hline \multirow{2}{*}{ 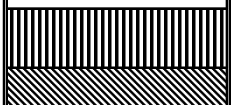 } & Muy común & $80-100 \%$ \\
\hline & Común & $61-80 \%$ \\
\hline \multirow{2}{*}{ 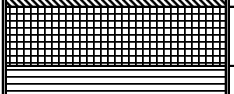 } & Frecuente & $41-60 \%$ \\
\hline & Ocasional & $21-40 \%$ \\
\hline & Raro & $0,1-20 \%$ \\
\hline & No & ntrado \\
\hline
\end{tabular}

día de muestreo.

\section{Aislamiento e identificación}

Las placas se incubaron a $25-28^{\circ} \mathrm{C}$ hasta 15 días, realizándose observaciones diarias a partir de las 48hrs. Los hongos filamentosos se identificaron a nivel de especie por sus características macro y micromorfológicas, mediante observaciones con lupa estereoscópica y preparaciones para microscopia con azul de lactofenol. Todos los hongos se reaislaron en agar papa dextrosa. Para la identificación de determinados géneros, se utilizaron medios de cultivo especiales como Nash Snyder, Czapek o Agar malta. Las colonias que no desarrollaron órganos de fructificación en los subcultivos tras 20 días de incubación a 25$28^{\circ} \mathrm{C}$, fueron agrupadas como micelio sin fructificación.

La presencia de los taxa fúngicos se contabilizó una sola vez en cada muestra, no importando si éste se repetía en la misma placa o aparecía en las otras dos.

Los hongos aislados se clasificaron en categorías en base a la frecuencia de aislamiento según el criterio propuesto por Yadav \& Madelin (12), (Tabla 1).

Para la clasificación taxonómica de los hongos se utilizaron las claves taxonómicas de: Nelson et al.(1983); Gams (1971); Onions et al. (1981); Von-Arx (1981); Hoog \& Guarro (2000); Samson et al. (2000.); Rifai (1969); Bissett (1991); Piontelli \& Toro (1994); Sivanesan (1987); Pitt(1986); Ellis \& Ellis (1987); Klich \& Pitt (1988); Carmichael et al.(1980) (13,14,15,16,17, 18,19,20,21,22,23, 24,25,26).

\section{RESULTADOS}

En los muestreos en primavera y en otoño en cada ciudad, se aislaron 571 colonias de hongos filamentosos de los que fueron identificados 35 géneros y 60 especies. La frecuencia de aislamiento de los hongos aislados discriminados por ciudad, estación, hora y Zona, se muestran en la tabla 2. Se encontraron hongos levaduri-formes pero para este estudio se consideraron únicamente los filamentosos.

La frecuencia de aislamiento de géneros por ciudades clasificados según Yadav y Madelin, se muestran en la tabla 3.

En Resistencia se aisló un mayor número de taxa (323) que en Corrientes (248), siendo más notoria esta diferencia en otoño. Asimismo, en la primera se obtuvo mayor diversidad de géneros (34/28).

De los 35 géneros encontrados, 7 (20\%) aparecen sólo en Resistencia: Scopulariopsis, Torula, Myriodontium, Clonostachys, Nigrospora, Chaetomiun y Stachybotrys. A diferencia de esto, Ulocladium fue hallado únicamente en Corrientes.

Los géneros recuperados exclusivamente en primavera, en una u otra ciudad, fueron:Chaetomium, Clonostachys, Mortierella, Chaetophoma y Ulocladium, mientras que, Myriodontium, Nigrospora, Stachybotrys, Syncephalastrum y Torula fueron recuperados sólo en otoño

Los géneros más representativos en número de taxa en ambas ciudades fueron: Asperguillus, con 9 especies y 84 aislamientos (15\% del total de especies); Penicillium con 5 especies, 46 aislamientos (8\%); Phoma con 5 especies y 43 aislamientos (7,5\%) y Fusarium con 5 especies y 39 aislamientos (6,8\%), todos estos, siempre mayoritarios en Resistencia (Tab.2).

Las 13 especies que se aislaron únicamente en Resistencia fueron: Aspergillus glaucus, Chaetomiun globosum, Clonostachys rosea, Colletotrichum coccodes, Fusarium graminearum, Myriodontium keratinophilum, Nigrospora sphaerica, Penicillium spinulosum, Phoma dennisii var. oculo hominis, Scopulariopsis brevicaulis, Stachybotrys chartarum, Torula herbarum y Trichoderma viride.

Las 7 especies que se aislaron únicamente en Corrientes fueron: Acremoniun kiliense, Aspergillus clavatus, Colletotrichum gloeosporioides, Fusarium subglutinans, Mucor indicus, Phoma glomerata, Ulocladium atrum.

En Resistencia, la frecuencia total de aislamientos no presentó diferencias entre otoño y primavera. Analizando las Zonas, no hay un patrón de recuperación en relación a las estaciones. En cambio según los horarios, aunque hubo poca diferencia, se obtuvo mayor número de aislamientos a mediodía que en las primeras horas de la mañana.

En Corrientes la frecuencia total de aislamientos fue notoriamente menor en otoño. El análisis por zonas no arrojó mayores diferencias, sin embargo, siempre fue menor en la Zona 1. En cambio, sí hay diferencia en relación al horario, se obtuvo mayor número de taxa al mediodía, lo cual fue más evidente en la Zona 1.

\section{DISCUSION}


Tabla 2: Frecuencia de aislamiento de hongos filamentosos discriminados por ciudad, estación, hora y zona.

\begin{tabular}{|c|c|c|c|c|c|c|c|c|c|c|c|c|c|c|c|c|}
\hline \multirow{3}{*}{$\begin{array}{c}\text { Ciudad } \\
\text { Estación } \\
\text { Zona } \\
\end{array}$} & \multicolumn{8}{|c|}{ Corrientes } & \multicolumn{8}{|c|}{ Resistencia } \\
\hline & \multicolumn{4}{|c|}{ primavera } & \multicolumn{4}{|c|}{ otoño } & \multicolumn{4}{|c|}{ primavera } & \multicolumn{4}{|c|}{ otoño } \\
\hline & \multicolumn{2}{|c|}{1} & \multicolumn{2}{|c|}{2} & \multicolumn{2}{|c|}{1} & \multicolumn{2}{|c|}{2} & \multicolumn{2}{|c|}{1} & \multicolumn{2}{|c|}{2} & \multicolumn{2}{|c|}{1} & \multicolumn{2}{|c|}{2} \\
\hline Hora & 1 & 2 & 1 & 2 & 1 & 2 & 1 & 2 & 1 & 2 & 1 & 2 & 1 & 2 & 1 & 2 \\
\hline Acremoniun kiliense Grutz & & 1 & & & 1 & 1 & 1 & & & & & & & & & \\
\hline Acremoniun strictum W.Gams & 1 & 1 & 1 & 1 & & 1 & 1 & & 1 & 1 & 2 & 2 & & & 1 & \\
\hline Alternaria alternata (Fr.) Keiss grupo & 2 & 2 & 2 & 3 & & & 2 & 3 & 3 & 4 & 1 & 3 & 1 & 1 & 2 & 3 \\
\hline Aspergillus clavatus Desm. & & 1 & 2 & & & & & & & & & & & & & \\
\hline Aspergillus fumigatus Fres. & & 1 & 1 & & & & & & & 2 & & & & & 1 & 2 \\
\hline Aspergillus glaucus (Mich. Fr.) Link & & & & & & & & & 1 & & 1 & 1 & & & & \\
\hline Aspergillus niger van Tieghem & & & 1 & 1 & & & 1 & & 2 & 1 & & & & & 1 & 1 \\
\hline Aspergillus ochraceus Wilhem & & & & 1 & & 1 & & & & & & & & 1 & 1 & \\
\hline $\begin{array}{l}\text { Aspergillus sydowii (Bain. \& Sart.) Thom \& } \\
\text { Church }\end{array}$ & 1 & & & & & & & & & & & & & 2 & & \\
\hline Aspergillus terreus Thom & & 1 & & & & & & & 1 & 1 & 1 & 1 & 1 & 1 & 1 & \\
\hline Aspergillus versicolor (Vuill.) Tiraboschi & & 2 & & & 1 & 2 & & & & & 2 & & 4 & 3 & 5 & 4 \\
\hline $\begin{array}{l}\text { Aureobasidium pullulans var. pullulans (de } \\
\text { Bary) Arn. }\end{array}$ & 1 & 1 & & 1 & & & & & & & & & 1 & & & \\
\hline $\begin{array}{l}\text { Bipolaris hawaiiensis (M.B. Ellis) Subram.\& } \\
\text { Jain }\end{array}$ & & 1 & 1 & 1 & & & & & 1 & & 1 & & & 1 & & \\
\hline \begin{tabular}{llll|} 
Botryiotrichum & piluliferum & Saccardo \& \\
Marchal & & \\
\end{tabular} & & & & 1 & & 1 & 1 & & & & 1 & & & & & \\
\hline Chaetomium globosum Kunze & & & & & & & & & & & 1 & & & & & \\
\hline Chaetophoma sp & & 1 & 1 & & & & & & 1 & & & & & & & \\
\hline Chrysonilia sitophila (Mont) v. Arx & 1 & & & & & 2 & 1 & & & & & 1 & & 1 & 3 & 2 \\
\hline Cladosporium cladosporioides (Fres.) de Vries & 5 & 6 & 6 & 6 & 3 & 5 & 5 & 6 & 5 & 5 & 5 & 5 & 4 & 6 & 5 & 5 \\
\hline Cladosporiun sphaerospermun Penz. & 1 & 2 & & & & 1 & & & & & 1 & & 1 & & 1 & 1 \\
\hline $\begin{array}{l}\text { Clonostachys rosea (Link:Fr.) Schroers, Samu- } \\
\text { els, Seifert \& W.Gams }\end{array}$ & & & & & & & & & & & & 1 & & & & \\
\hline Colletotrichum coccodes (Wallr.) Hughes & & & & & & & & & & & 1 & & 1 & & & \\
\hline Colletotrichum gloeosporioides (Penz.)Sacc. & & & & & & & 1 & & & & & & & & & \\
\hline Curvularia lunata (Wakker) Boedijn & & 2 & 3 & 3 & 1 & 2 & & & & 1 & & 4 & 1 & 2 & & 2 \\
\hline Doratomyces stemonitis (Pers.)Morton\&Smith & & & & & & & 1 & & & 1 & & & & & & \\
\hline Emericella nidulans (Eidam) Winters & & & & 1 & & 1 & 1 & & & & & & 3 & & 1 & 1 \\
\hline Epicoccum nigrum Link & & 1 & 1 & & & & & & 2 & 1 & 2 & 2 & 1 & 1 & & \\
\hline $\begin{array}{l}\text { Fusarium chlamydosporum Wollenw. \& } \\
\text { Reink. }\end{array}$ & & 1 & 1 & & & & & & 1 & 1 & 1 & 2 & & & & 2 \\
\hline Fusarium graminearum Schwabe & & & & & & & & & & 1 & & & & & & \\
\hline Fusarium oxysporum Schlecht. Fr. & 1 & & & 2 & 1 & 1 & 1 & 2 & 1 & 2 & 2 & & 2 & 1 & 1 & \\
\hline Fusarium solani (Mart.) Sacc. & & & 2 & & 1 & 2 & & 1 & 1 & 1 & & 1 & & & 1 & 1 \\
\hline Fusarium subglutinans Wollenw. \& Reink. & & & 1 & & & & & & & & & & & & & \\
\hline Harzia acremonioides (Harz) Cost. & & 1 & & & 2 & 2 & & & & & & 1 & & & & \\
\hline Leptosphaerulina trifolli (Rostrup) Petrak & 1 & 1 & 3 & & & & & & 2 & 2 & 1 & 0 & & & 1 & 0 \\
\hline
\end{tabular}


(Continuación Tabla 1)

\begin{tabular}{|c|c|c|c|c|c|c|c|c|c|c|c|c|c|}
\hline \multirow{3}{*}{$\begin{array}{c}\text { Ciudad } \\
\text { Estación } \\
\text { Zona } \\
\end{array}$} & \multicolumn{6}{|c|}{ Corrientes } & \multicolumn{7}{|c|}{ Resistencia } \\
\hline & \multicolumn{3}{|c|}{ primavera } & \multicolumn{3}{|c|}{ otoño } & \multicolumn{4}{|c|}{ primavera } & \multicolumn{3}{|c|}{ otoño } \\
\hline & 1 & \multicolumn{2}{|c|}{2} & 1 & \multicolumn{2}{|c|}{2} & \multicolumn{2}{|c|}{1} & \multicolumn{2}{|c|}{2} & 1 & \multicolumn{2}{|c|}{2} \\
\hline Hora & 1 & 1 & 2 & 1 & 1 & 2 & 1 & 2 & 1 & 2 & 1 & 1 & 2 \\
\hline Micelio sin fructificación & 5 & 5 & 5 & \begin{tabular}{l|l}
4 & 6
\end{tabular} & \begin{tabular}{l|l}
5 & 5
\end{tabular} & 6 & 4 & 6 & 5 & 4 & 4 & 3 & 6 \\
\hline Mortierella polycephala (Coemans) & & & 1 & & & & & 1 & 1 & & & & \\
\hline Mucor indicus Lendner & & & 1 & & & & & & & & & & \\
\hline Mucor racemosus Fr. & 1 & & & & & & & & & & & & 1 \\
\hline $\begin{array}{llll}\begin{array}{l}\text { Myriodontium } \\
\text { Polonelli }\end{array} & \text { keratinophilum } & \text { Samson \& } \\
\end{array}$ & & & & & & & & & & & 1 & & 1 \\
\hline Nigrospora sphaerica (Sacc.) Mason & & & & & & & & & & & & 1 & \\
\hline Penicillium brevicompactum Dierckx & 1 & 1 & 1 & & & & 1 & & & & & 1 & 1 \\
\hline Penicillium chrysogenum Thom & 1 & & & & & 1 & & & & 1 & & 2 & 1 \\
\hline Penicillium expansum Link ex S.F. Gray & \begin{tabular}{l|l}
1 &
\end{tabular} & & & & & & & 1 & & & & & \\
\hline Penicillium frequentans Westling & \begin{tabular}{l|l}
2 & 2 \\
\end{tabular} & 2 & 2 & & 1 & 2 & 3 & 1 & 2 & 2 & 1 & 2 & 2 \\
\hline Penicillium spinulosum Thom & & & & & & & & & 1 & & & & \\
\hline Pestalotiopsis sydowiana (Bres.) Sutton & 1 & & & & & & & & & & & 1 & \\
\hline Phoma cruris-hominis Punithalingam & 1 & & & 1 & & & & & & & 1 & & \\
\hline $\begin{array}{l}\text { Phoma dennisii var. oculo hominis (Pun.) } \\
\text { Boerema et.al. }\end{array}$ & & & & & & & & & & & & 1 & 1 \\
\hline Phoma eupyrena Sacc. & 1 & & 1 & 2 & & 1 & 2 & 1 & 1 & 1 & & & \\
\hline $\begin{array}{l}\text { Phoma glomerata (Corda) Wollenw.\& Hocha- } \\
\text { pfel }\end{array}$ & 1 & 1 & & & & & & & & & & & \\
\hline Phoma herbarum Wstend. & 1 & 1 & 1 & 1 & & 1 & 1 & 5 & 1 & 4 & 1 & 1 & 2 \\
\hline Rhizopus stolonifer (Ehrenb.: Fr.) Vuill. & 1 & & & 1 & 2 & 2 & & 1 & & 1 & 1 & 1 & \\
\hline Scopulariopsis brevicaulis (Sacc.) Bain. & & & & & & & & 1 & & & & 1 & \\
\hline Scytalidium dimidiatum Oyeka \& Gugnani & 1 & 1 & & 1 & & 1 & 1 & 2 & & & 1 & 1 & 2 \\
\hline Scytalidium hyalinum Campbell \& Mulder & 1 & & & & & & & 1 & 1 & & & 1 & \\
\hline $\begin{array}{l}\text { Stachybotrys chartarum (Ehrenb. ex.Link) } \\
\text { Hughes }\end{array}$ & & & & & & & & & & & 1 & & \\
\hline Syncephalastrum racemosum Cohn & & & & & & 1 & & & & & & & 1 \\
\hline Torula herbarum (Pers.) Link & & & & & & & & & & & & 1 & 1 \\
\hline Trichoderma harzianum Rifai & & 1 & 2 & & & & 3 & 1 & 1 & 3 & 2 & 3 & 2 \\
\hline Trichoderma viridae Pers. & & & & & & & 1 & 1 & & & & & \\
\hline Ulocladium atrum Preuss & & 1 & & & & & & & & & & & \\
\hline Verticillium tenerum Nees ex Pers. & & 1 & & & & & & 1 & 1 & & & & \\
\hline Total hora & \begin{tabular}{l|l}
29 & 43 \\
\end{tabular} & 41 & 35 & \begin{tabular}{|l|l}
15 & 33 \\
\end{tabular} & $3 \quad 24$ & 28 & 38 & 47 & 37 & 40 & \begin{tabular}{|l|l|l}
33 & 35 \\
\end{tabular} & 3942 & 247 \\
\hline Total zona & 72 & 7 & 6 & 48 & 5 & 2 & & 85 & 7 & 7 & 72 & & 89 \\
\hline Total estación & & 48 & & & 100 & & & 16 & 62 & & & 161 & \\
\hline Total Ciudad & & & 2 & & & & & & & 32 & 23 & & \\
\hline
\end{tabular}


Tabla 3: Frecuencia de aislamiento de géneros por ciudades clasificados según Yadav y Madelin.

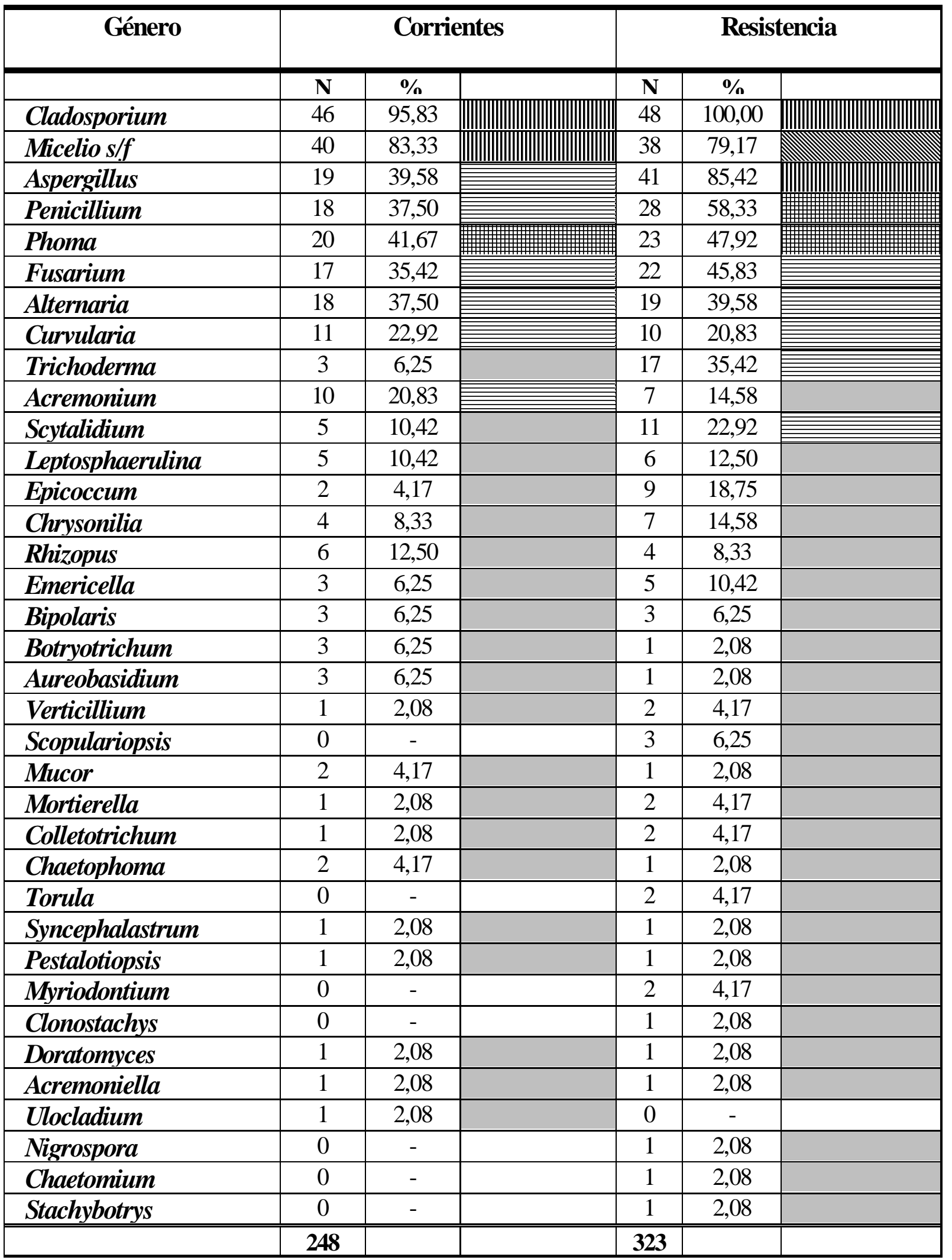


La interpretación de los resultados en muestreos de aire, es frecuentemente problemática por una multiplicidad de factores. A saber, el nivel de los propágulos aéreos varía considerablemente hora a hora o quizás momento a momento debido a las fluctuaciones de temperatura, humedad relativa, dirección y velocidad del viento. Además, la viabilidad de los propágulos fúngicos declina desde el momento en que son producidos porque la respiración celular agota las reservas endógenas (18) . Asimismo, los conidios de algunas especies son dañadas fácilmente por la luz o la desecación. También deben ser destacadas diferencias biológicas entre algunos géneros, por ejemplo, Aspergillus y Penicillium producen gran cantidad de conidios que fácilmente son aerotranspor-tadas, en cambio los conidios húmedos o las esporas producidas en cuerpos de fructificación sólo pueden ser detectadas cuando el material se seca y son liberadas al aire. Se ha demostrado también que los conidios de Asper-gillus y Penicillium sobreviven a la desecación por décadas (18). En contraste, las de Stachybotrys chartarum declinan su viabilidad rápidamente, por lo tanto, el aislamiento de esta especie de muestras de aire colectadas sobre medio de cultivo debe ser considerado de manera diferente del aislamiento de especies de Penicillium en la misma placa.

Por otro lado, algunos hongos no pueden ser detectados en medios de agar, aún cuando éste sea adecuado para su crecimiento, porque ellos compiten pobremente con otros hongos sobre el substrato o sus colonias no son suficientemente distintivas. Así, algunos autores opinan que Alternaria spp es subregistrada en las placas de agar de muestreo, pero como sus conidios son tan típicos que se reconocen facilmente al colectarse por captadores volumétricos. Sin embargo, en nuestra experiencia encontramos mayor porcentaje de Alternaria que el registrado en Porto Alegre y en West Bengal (India) donde trabajaron con muestreadores de aire $(27,28)$.

Asimismo, las grandes diferencias metodoló-gicas y geográficas registradas en la literatura aeromi-cológica dificultan la comparación de los resultados. Aún así, la diferencia en la carga fúngica del aire observada en Corrientes y Resistencia, cuando se realiza la comparación por estación, es coincidente con los datos bibliográficos que muestran diferencias en la composición de la micota aérea según las estaciones del año. En este caso, al relacionar el número de especies por estación se observo mayor cantidad en primavera que es la estación más cálida y seca de las estudiadas. Este resultado se debería a la acción de los vientos que actúan sobre el suelo seco, levantando el polvo y dispersando con él los propágulos de dispersión. Este hecho concuerda con lo informado por Burge et al. (29), que también encontraron alta prevalencia de propágulos fúngicos durante la estación cálida y seca, lo mismo que en las ciudades de Santiago de Chile y Porto Alegre $(9,27)$. Lo contrario fue registrado en la ciudad de Córdoba (Argentina) donde la mayor densidad se produjo en los meses más fríos (30).

El hecho de que en Corrientes se obseravran diferencias entre las frecuencias de aislamiento en los distintos horarios y en Resistencia no, podría explicarse por la fisiografía de las ciudades y el fenómeno de inversión de temperaturas. Este es el fenómeno por el cual se imposibilita el desplazamiento vertical normal del aire cálido más cercano a la superficie terrestre, favoreciendo la permanencia de los contaminates sólidos resuspendidos por el movimiento vehicular y de las personas. La situación se dramatiza en Resistencia pues su emplazamiento en la depresión de una planicie aluvial acentúa el mismo.

En estudios previos de micota en ambientes abiertos realizados en Resistencia, La Plata y Córdoba (Argentina) se destaca al género Alternaria como el más frecuentemente aislado, seguido de Penicillium, mientras que en nuestra experiencia, en las dos ciudades, fue Cladosporium el género predominante seguido por Aspergillus, considerado como de ambientes tropicales y subtropicales $(12,30,31)$. Esta diferencia, en el caso de Resistencia, podría deberse a que el muestreo fue realizado a nivel del suelo, según información personal del autor (12). En los otros estudios no se indica a que altura se expusieron las placas. Esta situación destaca, una vez más, las dificultades para la comparación de datos debido al uso de diferentes metodologías.

Es notoria la mayor frecuencia de aparición de especies de Aspergillus en relación a las de Penicillium, si bien diversos autores informan en general una preponderancia del segundo $(9,11,30,31)$. Esta diferencia es más llamativa en la ciudad de Resistencia, cosa que ya había sido notada en trabajos anteriores (12). La mayoría de las especies del género Aspergillus son importantes desde el punto de vista de la salud, se relacionan con alergias y enfermedades broncopulmonares. En esta región, donde existe una mediana prevalencia de tuberculosis, la posibilidad del desarrollo de aspergilosis intracavitaria es una complicación a ser considerada. Similares comentarios podrían aplicarse a Fusarium.

El número de géneros encontrados (35) en este estudio no difiere del hallado en muestreos realizados en diferentes ciudades del mundo, como Santiago de Chile 34 géneros, Milán (Italia) 24, West Bengal (India) 35 y Palencia (España) 34 géneros $(3,4,9,10,28)$. A diferencia de esto, sí se observa mayor número que el informado en estudios previos realizados en ciudades argentinas tales como Resistencia donde se hallaron 20 géneros, la Plata (B. Aires) 19 y Córdoba 24 géneros (12,30, 31).

Por los factores que afectan a los propágulos aéreos y su evaluación, se puede concluir que hay poca 
base sólida para comparar la cantidad de propágulos fúngicos obtenidos en un muestreo de aire, pero la proporción de diferentes tipos de hongos en unidades muestrales individuales, por depositación en placas de agar o a través de muestreadores, provee una información fidedigna (1) y por ello se recomienda la utilización de medios y métodos de muestreo combinados.

\section{REFERENCIAS}

1.- Samson, R.A.; Flannigan, B.; Flannigan, M.E.; Verhoeff, A.P.; Adan, O.C.G \& Hoekstra, E.S. (1994). Health implications of fungi in indoor environments. Air quality monographs- Vol. 2. Elseiver. Ámsterdam.

2.- Cappello, R.; Donovarros, C. \& Giono, S. (2003). La diversidad microbiana en México. http://www.conabio.gob.mx/institucion/ conabio_espanol/doctos/microbios.html

3.- Ibañez, V. \& Rojas, G. (1999). Aerosporas fúngicas del área oriente de Santiago - Chile (1996-1997). Boletín Micológico14: 83-89

4.- Picco, A.M. \& Rodolfi, M. (2000). Airborne fungi as biocontaminants at two Milan underground stations. International Biodeterioration \& Biodegradation. 45:43-47

5.- Antico, A. (2000). Environmental factors and allergic airways diseases. Aerobiología. 165:321-329

6.- Fluckiger, B.; Koller, T. \& Monn, C. (2000). Comparison of airborne spore concentrations and fungal allergen content. Aerobiología.16: 393-396

7.- Facultad De Humanidades Universidad Nacional del Nordeste. (1972). Geográfica $\mathrm{N}^{\circ} 1$. Revista del Instituto de Geografía. Resistencia. Argentina 1972; 8 - 13

8.- Bruniard, E.D. (1966). Bases fisiogeográficas para una división regional de la provincia de Corrientes. Revista Nordeste. 8:9-26

9.- Ibáñez, V.; Thompson, L. \& Mañalich, J. (1998). Fluctuación estacional de hongos anemófilos en Santiago- norte- Chile. Boletín Micológico. 13: 47-56

10.-Baudilio, M.; Fombelle, M.; Fernández, D. \& Valencia, R. (1996). Aerobiological study of fungal spores from Palencia (Spain). Aerobiología. 12:27-35

11.- Shadzi, S.; Zahraee, M.H. \& Chadegani, M. (1993). Incidence of airborne fungi in Isfahan, Iran. Mycoses. 36: $69-73$

12.- Mangiaterra, M.; Alonso, J.M.; Medina, E. \& Cerbera, L. (1993) . Microflora Anemófila de la ciudad de Resistencia. Revista Argentina de Micología. 16:10-16

13.- Onions, A.; Allsopp, D. \& Eggins, H. (1981). Smiths‘s introduction to industrial Micology, $7^{\text {a }}$ ed. John Wiley \& Sons. New York.

14.- Hoog, G. \& Guarro, J. (2000). Atlas of Clinical Fungi, $2^{\mathrm{a}}$ ed. The Netherlands: Centraalbureau voor Schimmelcultures. Baarn and Delft.

15.- Nelson, P.; Tousson, T.; Marasas, F. (1983). Fusarium species. An illustrated Manual for identification. The Pennsylvania State University Press. University Park and London.

16.- Gams, W. (1971). Cephalosporium-artige Schimmelpilze (Hyphomycetes). G Fisher Verlag. Stuttgart.

17.- Von-Arx, J.A. (1981). The genera of fungi sporulating in pure culture. $3^{\mathrm{a}}$ ed. Edit J. Crame. Vaduz.

18.- Samson, R.; Hoekstra, E.; Frisvad, J.C. \& Filtenborg, O. (2000) . Introduction to food and airborne fungi. Utrecht. Centraalbureau voor Shimmalcultures. The Netherlands.

19.- Rifai, M.A. (1969). A Revision of the Genus Trichoderma. Mycological Papers $\mathrm{N}^{\mathrm{o}} 116$

20.- Bissett, J. (1991). A Revision of the Genus Trichoderma. II. Infrageneric classification, Can. J. Bot. 69:2357 - 2372

21.- Piontelli, E. \& Toro, M.A. (1994). Manual de identificación para microhongos comunes en alimentos. Universidad de Valparaíso. Escuela de Medicina. Cátedra de Micología. Valparaíso.

22.- Sivanesan, A. (1987). Graminicolous species of Bipolaris, Curvularia, Drechslera, Exserohilum and their teleomorphs. Mycological papers, $\mathrm{N}^{\circ}$ 158. C.A.B. International Mycological Institute.

23.- Pitt, J.I. (1986). A laboratory guide to common Penicillium species, Reprinted. Australia: Commonwealth Scientific an d Industrial Research Organisation, Division of food Reserch.

24.- Ellis, M.B. \& Ellis, J.P. (1987). Microfungi on land plants. An identification handbook. Croom Helm. Australia.

25.- Klich, M.A. \& Pitt, J.I. (1988). A laboratory guide to common Aspergillus species and their teleomorphs. Reprinted. Commonwealth Scientific and Industrial Research Organization, Division of food Processing. Australia.

26.- Carmichael, J.; Kendrick, B.; Conners, I.; Sigler, L. (1980). General of Hyphomycetes. The University of Alberta Press. Canada.

27.- Mezzari, A.; Perin, C.; Santos Júnior, S.A.; Bernd, L. (2002). Airborne fungi in the city of Porto Alegre, Rio Grande do Sul, Brazil. Rev. Inst. Med Trop. S. Paulo. 44:269-272

28.- Adhikari, A.; Sen, M.; Gupta-Bhattacharya, S.; Chanda, S. (2000) . Incidence of allergenically significant fungal aerosol in a rural bakery of West Bengal, India Mycopathologia. 149: 35-45

29.- Burge, H.; Leventin, E.; Muilenberg, M.; Solomon, W.R. (1997) . Fungus spores identification. Asthma and Immunology. 322

30.- Quiroga de Pascual, R.L. \& Nobile, R. (1985). Incidencia de hongos ambientales durante un año en la ciudad de Córdoba. Rev. Arg. de Micol. 8:16-22

31.- Iovannitti,C. \&Tiraboschi, I.N. (1985). Hongos anemófilos 